\title{
BOUNDARY BEHAVIOR OF SOLUTIONS OF PARABOLIC EQUATIONS WITH DISCONTINUOUS COEFFICIENTS ${ }^{1}$
}

\author{
BY NEIL A. EKLUND ${ }^{2}$
}

Communicated by James Serrin, March 31, 1971

Introduction and results. Consider the parabolic operator in divergence form

$$
L u=u_{t}-\left\{a_{i j}(x, t) u_{x_{i}}\right\}_{x_{j}}
$$

where we have employed the convention of summation over repeated indices. Here $x=\left(x_{1}, x_{2}, \cdots, x_{n}\right)$ denotes a point in $E^{n}$ with $n \geqq 1$ and $t$ denotes a point on the real line. Assume that the coefficients of (1) are bounded measurable functions of $(x, t)$ in $S=E^{n} \times(-1,2 T)$ for some $T>0$ and that there is a constant $\lambda>0$ such that $a_{i j}(x, t) z_{i} z_{j} \geqq \lambda|z|^{2}$ almost everywhere in $S$ for all $z \in E^{n}$.

Let $G$ be a bounded domain in $E^{n} \times(0, T)$. We prove that to each function $f \in C(\partial G)$ there corresponds a weak solution $u$ of the boundary value problem

$$
\begin{aligned}
L u & =0, & & \text { in } G, \\
u(x, t) & =f(x, t), & & \text { on } \partial G .
\end{aligned}
$$

The precise definition of weak solution is given below; here it suffices to know that $u \in C(G)$. The notion of regularity of a boundary point is defined in the usual way: a point $(y, s) \in \partial G$ is said to be regular for $L$ in $G$ if, for each $f \in C(\partial G)$, the corresponding solution $u$ of (2) satisfies

$$
\lim _{(x, t) \rightarrow(y, s) ;(x, t) \in G} u(x, t)=f(y, s) .
$$

D. G. Aronson [1] has shown that every operator of the form (1) possesses a fundamental solution $g(x, t ; \xi, \tau)$ and this fundamental solution satisfies

A MS 1970 subject classifications. Primary 35K20, 35K10; Secondary 35R05.

Key words and phrases. Parabolic equations, divergence structure, boundary regularity.

1 This research was partially supported by the United States Air Force Office of Scientific Research under Contract No. AF-AFOSR 883-67 at the University of Minnesota.

2 The results in this paper are taken from the author's doctoral dissertation written under the supervision of Professor D. G. Aronson and presented at the University of Minnesota, August 1970. The proofs will appear in full elsewhere. 


$$
C_{1} g_{1}(x-\xi, t-\tau) \leqq g(x, t ; \xi, \tau) \leqq C_{2} g_{2}(x-\xi, t-\tau)
$$

in $S$ for $t>\tau$, where $g_{i}$ is the fundamental solution of the heat operator $L_{i} u=u_{t}-\alpha_{i} \Delta u$, and where $\alpha_{i}$ and $C_{i}$ are positive constants, $i=1,2$. The purpose of this note is to announce the following result which relates regularity of boundary points for $L$ to an ordering of fundamental solutions exemplified by (3).

THEOREM. Let $L_{1}$ and $L_{2}$ be operators of the form (1) and assume their fundamental solutions $g_{1}, g_{2}$ satisfy

$$
g_{1}(x, t ; \xi, \tau) \leqq K g_{2}(x, t ; \xi, \tau)
$$

in $S$ for some constant $K$. If $(y, s) \in \partial G$ is regular for $L_{1}$ in $G$, then $(y, s)$ is regular for $L_{2}$ in $G$.

For uniformly elliptic equations in divergence form, Littman, Stampacchia, and Weinberger [3] have shown that the regularity or irregularity of a boundary point is independent of the equation. Therefore, to classify a given boundary point it suffices to consider only Laplace's equation. The following corollary to our theorem gives an analogous result for equations of the form (1) in a cylindrical domain.

Corollary. Suppose $G=Q \equiv \Omega \times(0, T]$, where $\Omega \subset E^{n}$ is a bounded domain. A point $(y, s) \in \partial \Omega \times(0, T]$ is regular for $L$ if and only if $y \in \partial \Omega$ is a regular point for Laplace's equation in $\Omega$.

The special case in which (1) is the equation of heat conduction was proved by Babuška and Výbroný [2]. Using the estimate (3) together with our theorem, the general result is an easy consequence of the special case.

For general domains in $E^{n+1}$, however, there is no uniformity of regular points for parabolic equations of the form (1). This follows from Petrovsky's work [4] on the equation of heat conduction in one space dimension. Moreover, using the results in [4] one can easily construct examples which show that our result is sharp.

Outline of the proof. The proof proceeds in two stages. We first prove the theorem when $G=Q$, a cylinder. The result for the cylindrical case is then used to derive the theorem in the general case via an approximation technique.

Cylindrical case. If the boundary values $f$ are sufficiently smooth it is easy to show that the boundary value problem (2) has a unique weak solution $u$ such that 


$$
u-f \in L^{\infty}\left[0, T ; L^{2}(\Omega)\right] \cap L^{2}\left[0, T ; H_{0}^{1,2}(\Omega)\right] .
$$

Using some extensions of the estimates derived in [1] and following the method of [3] there is a natural extension of this solution operator to $C(\partial Q)$. The function $u$ obtained in this manner is called the weak solution of the boundary value problem and has the following properties:

(a) for each $\delta>0, u(x, t) \in L^{2}\left[\delta, T ; H_{\mathrm{loc}}^{1,2}(\Omega)\right] \cap L^{\infty}\left[\delta, T ; L_{\mathrm{loc}}^{2}(\Omega)\right]$;

(b) $\iint_{Q}\left[a_{i j} u_{x_{i}} \phi_{x_{j}}-u \phi_{t}\right] d x d t=0$ for all $\phi \in C^{1}(Q)$ vanishing in a neighborhood of $\partial Q$;

(c) there is a sequence $\left\{f^{N}\right\}$ of polynomials on $\partial Q$ converging to $f$ in the supremum norm such that the sequence of solutions $\left\{u^{N}\right\}$ of the boundary value problems

$$
\begin{aligned}
L u & =0, & & \text { in } Q, \\
u(x, t) & =f^{n}(x, t), & & \text { on } \partial Q,
\end{aligned}
$$

converges weakly in $L^{2}\left[0, T ; H^{1,2}(\Omega)\right]$ to $u$.

Note that points in $\Omega \times(T)$ are always irregular and points in $\Omega \times(0)$ are always regular. Therefore, we need only consider points of $\partial \Omega \times(0, T]$. Moreover, since $u\left(x_{0}, t_{0}\right)$ depends on the boundary data $f(x, t)$ for $t<t_{0}$, we need only consider $f \in C\left(\partial_{P} Q\right)$ where $\partial_{P} Q$ denotes the parabolic boundary of $Q, \partial Q-\{\Omega \times(T)\}$.

A function $V(x, t)$ is a barrier for $(y, s) \in \partial \Omega \times(0, T]$ with respect to $L$ if

(a) $V(x, t) \in L^{\infty}\left[0, s ; L^{2}(\Omega)\right] \cap L^{2}\left[0, s ; H^{1,2}(\Omega)\right]$,

(b) $V$ is a weak solution of $L u=0$ in $\Omega \times(0, s]$,

(c) for any $\rho>0$ there is an $m>0$ such that

$$
V \geqq m \text { on }\left\{\partial_{P} Q \cap[0, s]\right\}-\{|x-y|<\rho, 0 \leqq s-t<\rho\} \text {, }
$$

(d) $V$ is continuous at $(y, s)$ with

$$
\lim _{(x, t) \rightarrow(y, s) ;(x, t) \in \Omega \times(0, s]} V(x, t)=V(y, s)=0 .
$$

As expected the regularity of a boundary point $(y, s)$ is equivalent to the existence of a barrier for $(y, s)$ with respect to $L$. The existence of a barrier is, in turn, equivalent to the continuity at $(y, s)$ for all sufficiently small $\rho>0$ of the solution $u^{\rho}$ of the exterior boundary value problem

$$
\begin{aligned}
L u & =0, \text { in } \mathfrak{C}\{x \in \mathcal{C} \Omega ;|x-y|<\rho\} \times(s-\rho, s], \\
u(x, s-\rho) & =0, \text { on } \mathfrak{e}\{x \in \mathcal{C} \Omega ;|x-y|<\rho\}, \\
u(x, t) & =1, \text { on } \partial\{x \in \mathcal{C} \Omega ;|x-y|<\rho\} \times[s-\rho, s] .
\end{aligned}
$$


We obtain the explicit representation

$$
u^{\rho}(x, t)=\int g(x, t ; \xi, \tau) d \nu^{\rho}(\xi, \tau)
$$

where $g$ is the fundamental solution of the operator $L$ and $\nu^{\rho}$ is a nonnegative Borel measure of bounded variation. The analogous representation in the elliptic case is derived by a variational argument [3]. This argument has no counterpart for parabolic equations and consequently we are required to obtain (5) directly from the differential equation by use of an approximation argument. The theorem in the cylindrical case follows from (4) and from the representation (5) by a modification of the argument used in [3].

General case. Let $\left\{t_{j}\right\}$ be a partition of $[0, T] . D=\cup_{j=1}^{a} \Omega_{j} \times\left(t_{j-1}, t_{j}\right]$ is called a multicylinder if each $\Omega_{j}$ is a finite union of smooth domains in $E^{n}$ and $D$ is connected. For the multicylinder $D$, define

$$
L^{2}\left[D ; H^{1,2}\right]=\bigcup_{j=1}^{a} L^{2}\left[t_{j-1}, t_{j} ; H^{1,2}\left(\Omega_{j}\right)\right] .
$$

The sequence $\left\{P^{N}\right\}$ of multicylinders is said to approximate $G$ if $\bar{P}^{N} \subset G, P^{N} \subset P^{N+1}$ for all $N \geqq 1$, and $P^{N} \rightarrow G$ as $N \rightarrow \infty$. Since $P^{N} \nearrow G$ it follows that $L^{2}\left[P^{N} ; H^{1,2}\right] \searrow$ and we define

$$
\operatorname{HC}(G)=\bigcap_{N=1}^{\infty} L^{2}\left[P^{N} ; H^{1,2}\right] .
$$

$u$ is said to be in $\mathfrak{K C}_{\mathrm{loc}}(G)$ if $u \in \mathcal{H}\left(G^{*}\right)$ for any domain $G^{*}$ whose closure is a compact subset of $G$.

Let $f \in C(\partial G)$ and extend $f$ to $F \in C(\bar{G})$. It follows from the cylindrical case that there is a solution $u^{N}$ of the boundary value problem

$$
\begin{aligned}
L u & =0, & & \text { in } P^{N}, \\
u(x, t) & =F(x, t), & & \text { on } \partial_{P} P^{N} .
\end{aligned}
$$

We define $u \in \mathcal{K}_{\text {loc }}(G)$ to be a weak solution of the boundary value problem

$$
\begin{aligned}
L u & =0, \quad \text { in } G, \\
u(x, t) & =f(x, t) \in C(\partial G)
\end{aligned}
$$

if (a) $u$ is a weak solution of $L u=0$ in $G$ and

(b) there is a sequence $\left\{P^{N}\right\}$ of multicylinders approximating $G$ and a continuous extension $F$ of $f$ to $\bar{G}$ for which a subsequence of the 
sequence $\left\{u^{N}\right\}$ of solution of the boundary value problems (6) converges weakly in $\mathfrak{H C}(G)$ to $u$.

As in the cylindrical case the regularity question reduces to the question of continuity of the function $u^{\rho}$. We use the multicylindrical approximation to obtain a representation formula for $u^{\rho}$ and then proceed as in the cylindrical case to prove the theorem.

\section{REFERENCES}

1. D. G. Aronson, Non-negative solutions of linear parabolic equations, Ann. Scuola Norm. Sup. Pisa (3) 22 (1968), 607-694.

2. I. Babuška and R. Výborny, Reguläre und stabile Randpunkte für das Problem der Wärmeleitungsgleichung, Ann. Polon. Math. 12 (1962), 91-104. MR 34 \#6346.

3. W. Littman, G. Stampacchia and H. F. Weinberger, Regular points for elliptic equations with discontinuous coefficients, Ann. Scuola Norm. Sup. Pisa (3) 17 (1963), 43-73. MR 28 \#4228.

4. I. Petrovsky, Zur ersten Randwertanfgabe der Wärmeleitungsgleichung, Compositio Math. 1 (1934), 383-419.

Vanderbilt University, Nashville, Tennessee 37203 